\title{
e-Migrinter
}

15 | 2017

Migrinter a trente ans : analyses et portraits

\section{Le Réseau Migrations en vidéo : les coulisses d'un projet collectif}

\section{Celio Sierra-Paycha}

\section{(2) OpenEdition \\ Journals}

Édition électronique

URL : https://journals.openedition.org/e-migrinter/854

DOI : 10.4000/e-migrinter.854

ISSN : 1961-9685

Éditeur

UMR 7301 - Migrinter

Référence électronique

Celio Sierra-Paycha, "Le Réseau Migrations en vidéo : les coulisses d'un projet collectif », e-Migrinter [En ligne], 15 | 2017, mis en ligne le, consulté le 20 mai 2021. URL : http://journals.openedition.org/emigrinter/854; DOI : https://doi.org/10.4000/e-migrinter.854

Ce document a été généré automatiquement le 20 mai 2021.

Tous droits réservés 


\title{
Le Réseau Migrations en vidéo : les coulisses d'un projet collectif
}

\author{
Celio Sierra-Paycha
}

1 Le projet que nous allons vous présenter a germé dans nos esprits au début de l'année 2016. Martine Brouillette et Marine Bertrand avaient été contactées par des étudiants de l'école européenne supérieure de l'image (EESI), une école d'art de Poitiers. Le thème des migrations, qui occupait déjà pleinement l'actualité médiatique, intéressait ses étudiants artistes... Une collaboration entre notre laboratoire et l'école d'art paraissait prometteuse. Nous souhaitions éventuellement nous engager autour d'un projet commun de représentation "artistique" ou "esthétique" de la dynamique réticulaire de nos activités de recherche, qu'il s'agisse de nos terrains, de nos lieux de vie, mais aussi de nos thématiques de travail. Une petite délégation de l'EESI vint nous visiter dans nos beaux locaux de la Maison des Sciences de l'Homme et de la Société (MSHS). Bien que cette rencontre n'aboutît pas sur un projet viable que l'on puisse vous présenter aujourd'hui, l'architecture, les transparences, les perspectives, les différents niveaux de cette bâtisse pittoresque, suscitèrent un fort enthousiasme chez nos confrères.

Hélas, pris par leurs examens de fin d'année, nos camarades ne pouvaient se libérer pour ce colloque, mais leur rencontre et nos échanges aiguisèrent notre sensibilité artistique. Sous les auspices des muses de l'art, frappés par la grâce d'Héphaïstos et d'Athéna, nous nous engageâmes nous-mêmes à produire quelque chose, encore peu identifié à l'époque, un produit fini en tout cas, qui porterait autour de la dynamique réticulaire de nos activités doctorales, avec une touch(e) arty, insufflée par l'énergie créatrice des étudiants de l'EESI.

3 Ayant, pour les plus anciens d'entre nous, pris part activement à la genèse du Réseau Migrations, dont tous les doctorants et docteurs sans poste de Migrinter sont membres, nous souhaitâmes alors rendre compte de l'émergence et de l'évolution de ce réseau, dont nous furent les témoins et les acteurs, et pour certaines parmi nous, des actrices très investies.

4 Encore fallait-il être capable de se représenter et de parler de cette dynamique. Nous disposions alors d'un certain nombre de données, liées au formulaire d'inscription en 
ligne des membres du réseau. Pour s'inscrire, un nouveau membre devait renseigner entre autres son sujet de thèse, de recherche postdoctorale, ou son mémoire de master, autant de data, de big data textuelles, qu'il était possible d'exploiter, grâce à un logiciel d'analyse statistique textuelle sous forme de "nuages de mots", et de "graphes de cooccurences" des termes les plus employés.

Mais les données que nous avions, ne suffisaient pas à rendre compte de l'implantation spatiale de nos différentes activités, ni des différentes caractéristiques des membres du réseau. Aussi, avons-nous songé à réaliser nous-mêmes notre propre enquête auprès des autres membres. Nous réalisâmes, alors une véritable enquête sociodémographique en ligne, dans les règles de l'art, avec une enquête pilote pour tester le premier questionnaire, protocole d'anonymisation, plusieurs relances pour diminuer l'attrition, apurement des données, constitution d'un système d'information géographique à l'échelle de la France métropolitaine et à l'échelle de la mappemonde. Après une première analyse statistique et cartographique de notre vaste échantillon de 47 enquêtés, nous jugeâmes nécessaires de dépasser le cadre habituel, impersonnel, de nos productions classiquement scientifiques et académiques, pour passer à un stade supérieur de la création.

6 L'équipe de doctorants structurée autour du projet ne manquait d'ailleurs pas de talent. Je me dois ici de faire une parenthèse sur le contexte résidentiel de mise en œuvre de ce projet. Notre principale salle de réunion de travail était le point de jonction d'un réseau internet éphémère reliant Tbilissi, Tel-Aviv, Alger, Poitiers, Marseille, Nantes, Montreuil-sous-Bois, par un rendez-vous SKYPE d'abord mensuel, puis hebdomadaire. Sous les auspices de la fibre optique et de la $4 \mathrm{G}$, nous échangeâmes lors de nombreux rendez-vous multi-situés, à l'image de nos terrains et de nos modes de vie.

7 Concernant les différents talents, il y eut d'emblée un élan créateur portée par les cartographes de l'équipe, pour rendre nos cartes plus personnelles, plus "arty". Un atelier de décalcomanie animée par Lucie Bacon, vit le jour à Poitiers, demandant plus de travail et de rigueur que de la peinture sur pierre de lave émaillée. Cet atelier fit des émules auprès de Martine Brouillette, Marine Bertrand et Maria Grazia Montella. D'autres se spécialisèrent dans l'animation visuelle, nous racontant par des "storymaps" l'évolution des activités du réseau migrations. Paloma Maquet et Amandine Desille, dévouées à l'art de l'infographie, auraient pu, si elles avaient connu les années 1970, réaliser la pochette d'un album d'un groupe de rock psychédélique.

8 Mais ce n'était pas là le fin mot de l'histoire. Comment rendre compte de cette animation visuelle? C'est là que les compétences de scénariste, monteuse, cinéaste, régisseuse de Julie Lemoux, Amandine Desille et Paloma Maquet furent mises au jour. Nous étions alors parés nous aussi pour passer le diplôme de l'EESI.

Bon visionnage !

Ce média ne peut être affiché ici. Veuillez vous reporter à l'édition en ligne http:// 


\section{AUTEUR}

\section{CELIO SIERRA-PAYCHA}

Doctorant en Géographie et Démographie, Migrinter - UMR 7301, CNRS / Université de Poitiers et INED, Paris

celio.sierra@gmail.com 